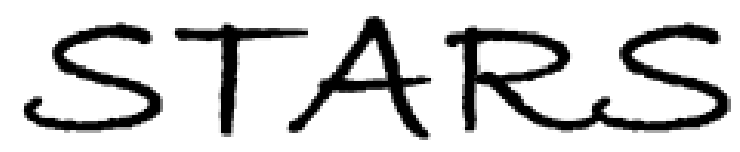

University of Central Florida

STARS

$1-1-2007$

\title{
Fabrication of periodic microstructures on flexible polyimide membranes
}

D. J. Shelton

University of Central Florida

J. S. Tharp

University of Central Florida

G. Zummo

University of Central Florida

W. R. Folks

University of Central Florida

G. D. Boreman

University of Central Florida

Find similar works at: https://stars.library.ucf.edu/facultybib2000

University of Central Florida Libraries http://library.ucf.edu

This Article is brought to you for free and open access by the Faculty Bibliography at STARS. It has been accepted for inclusion in Faculty Bibliography 2000 s by an authorized administrator of STARS. For more information, please contact STARS@ucf.edu.

\section{Recommended Citation}

Shelton, D. J.; Tharp, J. S.; Zummo, G.; Folks, W. R.; and Boreman, G. D., "Fabrication of periodic microstructures on flexible polyimide membranes" (2007). Faculty Bibliography 2000s. 7640.

https://stars.library.ucf.edu/facultybib2000/7640

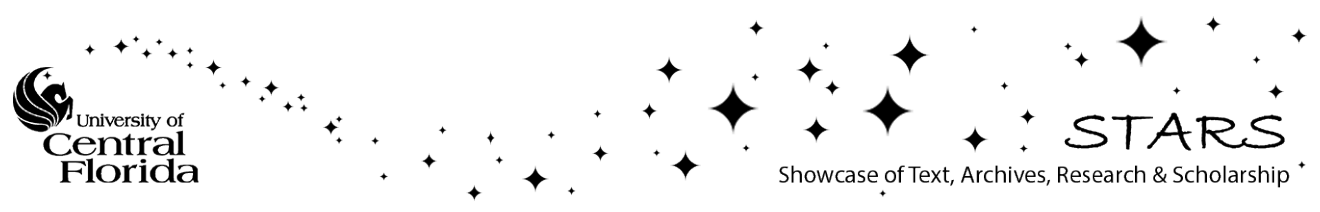




\section{Fabrication of periodic microstructures on flexible polyimide membranes}

D. J. Shelton, J. S. Tharp, G. Zummo, W. R. Folks, and G. D. Boreman

Citation: Journal of Vacuum Science \& Technology B: Microelectronics and Nanometer Structures Processing, Measurement, and Phenomena 25, 1827 (2007); doi: 10.1116/1.2794054

View online: https://doi.org/10.1116/1.2794054

View Table of Contents: https://avs.scitation.org/toc/jvn/25/6

Published by the American Institute of Physics

\section{ARTICLES YOU MAY BE INTERESTED IN}

The physics of metal oxide varistors

Journal of Applied Physics 46, 1332 (1975); https://doi.org/10.1063/1.321701

Strain and surface morphology in lattice-matched $\mathrm{ZnSe} / \mathrm{In}_{x} \mathrm{Ga}_{1-\mathrm{X}} \mathrm{As}$ heterostructures

Journal of Applied Physics 83, 2504 (1998); https://doi.org/10.1063/1.367011

Transducer Design for Ultrasonic Delay Lines

The Journal of the Acoustical Society of America 27, 302 (1955); https://doi.org/10.1121/1.1907519

Phase-locked oscillator optimization for arrays of Josephson junctions

Journal of Applied Physics 76, 3835 (1994); https://doi.org/10.1063/1.357387

Plasma diffusion from a low pressure radio frequency source

Journal of Vacuum Science \& Technology A 9, 661 (1991); https://doi.org/10.1116/1.577385

Thin-film resistor fabrication for InP technology applications

Journal of Vacuum Science \& Technology B: Microelectronics and Nanometer Structures Processing,

Measurement, and Phenomena 20, 871 (2002); https://doi.org/10.1116/1.1473179 


\title{
Fabrication of periodic microstructures on flexible polyimide membranes
}

\author{
D. J. Shelton, ${ }^{\text {a) }}$ J. S. Tharp, G. Zummo, W. R. Folks, and G. D. Boreman \\ College of Optics and Photonics/CREOL, University of Central Florida, Orlando, Florida 32816-2700
}

(Received 22 June 2007; accepted 12 September 2007; published 15 October 2007)

\begin{abstract}
Periodic metallic microstructures were fabricated on polyimide membranes. Techniques were developed to maintain flatness of the membrane during processing while still allowing for flexibility in the final structure. For proper functionality of the structures, it was necessary to first fabricate a continuous metallic film and a continuous dielectric layer on top of the flexible substrate, which underlaid the periodic microstructure. Flexibility of the overall structure was maintained by using a polymer as the dielectric layer, which was constrained to have high optical transmission over the infrared wavelength range of 6-14 $\mu \mathrm{m}$. Three candidate polymers were evaluated, and their measured optical properties are presented. Benzocyclobutene was found to be the best choice for this application. The final structure fully populated a $10 \mathrm{~cm}$ (4 in.) diameter flexible membrane with microstructures of excellent uniformity. (C) 2007 American Vacuum Society.
\end{abstract}

[DOI: $10.1116 / 1.2794054]$

\section{INTRODUCTION}

Microlithography techniques used in the fabrication of photonic devices traditionally use rigid substrates such as $\mathrm{Si}$ wafers. However, there exist specific applications for devices such as infrared (IR) detector arrays and IR frequency selective surfaces (FSS) which require flexible substrates, so that the final structure is able to be conformally contacted to a nonplanar surface. The conformal substrate considered in this study was the HD Microsystems liquid polyimide.

FSS elements appropriate for the IR (Ref. 1) typically have critical dimensions between 0.2 and $2 \mu \mathrm{m}$, with excellent spatial uniformity of the final periodic structure required. The FSS shown in Fig. 1 consists of three layers on top of the underlying structural substrate, a continuous metallic ground plane, a continuous dielectric standoff layer, and the periodic microstructural FSS elements. The thickness of the standoff layer is typically $\lambda / 4 n$, where $\lambda$ is the center of the design wavelength band of operation and $n$ is the refractive index of the standoff layer, which yields thicknesses in the range of a few micrometers. The ground plane and FSS elements are fabricated from metallic films of typical thickness of $0.1-0.2 \mu \mathrm{m}$. The Jerusalem cross designs were used in this study as the unit cell geometry for the FSS (compared to the square loop designs of Ref. 1) because of the presence of a second harmonic resonance that was useful in applications such as frequency selective surface filters. ${ }^{2}$

\section{MATERIAL SELECTION AND PROPERTIES}

When fabricating on a flexible substrate, it is desirable that the tensile moduli of the substrate and standoff layer be similar, which indicates that polymers are considered as candidate materials. For example, HD Microsystems liquid polyimide and benzocyclobutene (a candidate polymer standoff layer) have moduli of 2.7 and $2.9 \mathrm{GPa}$, respectively. Standoff materials used previously ${ }^{1}$ had tensile moduli rang-

\footnotetext{
${ }^{a)}$ Electronic mail: dshelton@ @reol.ucf.edu
}

ing from $80 \mathrm{GPa}$ for $a$-Si to $223 \mathrm{GPa}$ for zirconia. However, choice of the standoff layer material is constrained by two considerations that tend to exclude most polymers. To maintain proper functionality of the FSS, it is desirable to minimize absorption losses in the standoff layer. It is also difficult to fabricate many common polymers with the quarter-wave thickness required. Thus, the polymer chosen for the standoff layer must have high IR transparency as being capable of being spun or rolled out to the desired thickness with good uniformity.

The high resolution required in the fabrication of the FSS elements places constraints on the flatness and surface roughness of the combined underlying structure of the substrate, ground plane, and standoff layer. Typical flexible substrates such as polyimide sheets are both locally rough and difficult to secure in a sufficiently flat configuration. Previous research $^{3,4}$ has addressed this issue by using liquid polyimide, which gives the user control over both the surface roughness and flatness of the polyimide substrate. However, the lithographic-resolution limit seen in these references was on the order of tens of micrometers, and relatively small areas of the substrate were used for the fabrication.

In this study, a full $10 \mathrm{~cm}$ (4 in.) diameter substrate was populated with FSS elements. The combination of large write area and small element size required that the substrate had less than $10 \mathrm{~nm}$ rms surface roughness, with a high degree of planarization.

To investigate the optical properties of the candidate polymers, a J.A. Woollam IR ellipsometer was used. The ellipsometer uses polarization data such as the amplitudes of the electrical field components and their relative phase shifts to determine the complex index of refraction by fitting to an appropriate model. To facilitate fabrication, three resists and spin-on dielectrics [ZEP, polydimethylglutarmide (PMGI), and benzocyclobutene (BCB)] were investigated to find standoff layer materials that met the optical and mechanical requirements. Zeon Chemicals ZEP 520A7 is a positive tone electron-beam resist that has favorable optical properties in 
(a)

Cross Section

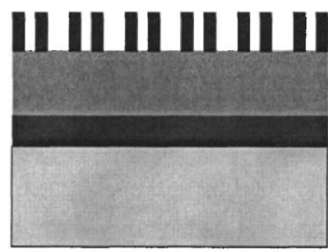

SS ERements

Standoff Layer

Ground Plone

Flexible Substrate (b)

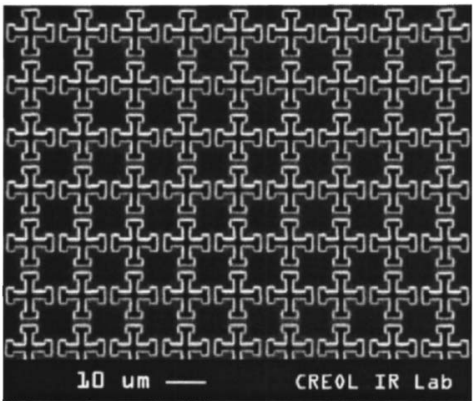

FIG. 1. FSS structural schematic with (a) cross section and (b) top view. the mid-IR, as shown in Fig. 2. ZEP can be difficult to fabricate on because of its poor adhesion to most metals. An adhesion promoter such as hexamethyldisilazane (HMDS) can improve adhesion to some oxides, but fabricating small metallic elements onto ZEP remains a challenge.

A complete fabrication process was attempted with polydimethlglutarmide (PMGI). MicroChem PMGI SF7 was used. PMGI is from the same family of polymers as polymethylmethacrylate (PMMA) and shares many of the same optical and chemical properties. As seen in Fig. 2, the loss associated with PMGI in the IR is significant. In two passes of $10.6 \mu \mathrm{m}$ radiation through a quarter-wave standoff layer (1.75 $\mu \mathrm{m}$ thickness), $44 \%$ of the radiation is absorbed. This leads to undesirable increases in emissivity beyond $10 \mu \mathrm{m}$ in PMGI.

PMGI and PMMA are both cured at $180{ }^{\circ} \mathrm{C}$, which is a lower cure temperature than the spin-on dielectrics require. PMGI is preferable over PMMA because it is not removed by solvents commonly used in postexposure lithographic processing such as acetone or xylene. PMGI also has this advantage over ZEP which is attacked by methelyne chloride and acetone over time. ${ }^{5}$

\section{FABRICATION TESTS FOR FEASIBILITY}

Using the PMGI standoff layer, problems were encountered in the lift-off of the excess metal between structures, after the FSS pattern had been exposed in photoresist and metallized with Ti. Figure 3 shows the boundary between

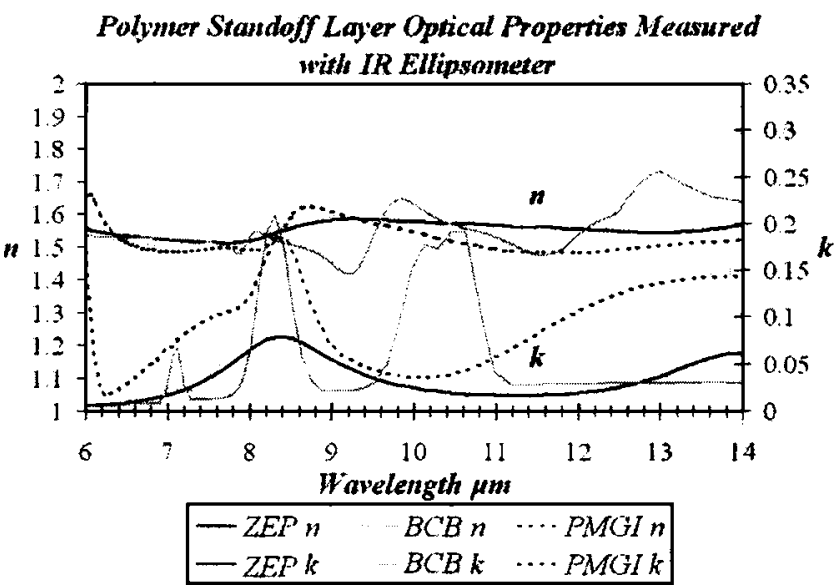

FIG. 2. Mid-IR optical properties of polymers. successfully lifted off structures, nonlifted off structures, and the region where the metal elements lost adhesion to the PMGI. The entire wafer was exposed, developed, and metallized. The lift-off was stopped before completion because the individual FSS elements were disassociating from the wafer in the solvent. The elements did not appear to lift-off immediately. Indeed, the small region of normally lifted off elements shows that there was some time between proper liftoff and disassociation of the elements, which unfortunately was not spatially uniform. The disassociated elements left behind ghost images that were measured via profilometry to be $20 \mathrm{~nm}$ deep.

The disassociation of the antenna elements and the appearance of ghost images appeared consistently over six separate trials without significant deviation. We suspect that this phenomenon was caused by the oxidation reaction between the polymer and the Ti elements. Normally, an oxidation reaction between a metal and a polymer is favorable and provides an adhesion mechanism. For polymers from the PMMA family, the new oxide species that forms after the reaction is not chemically bonded to the remainder of the polymer. During the reaction, thermal energy is transferred from the metal elements to the PMGI beneath causing an amorphization of the polymer carbon structure. ${ }^{6}$ This re-

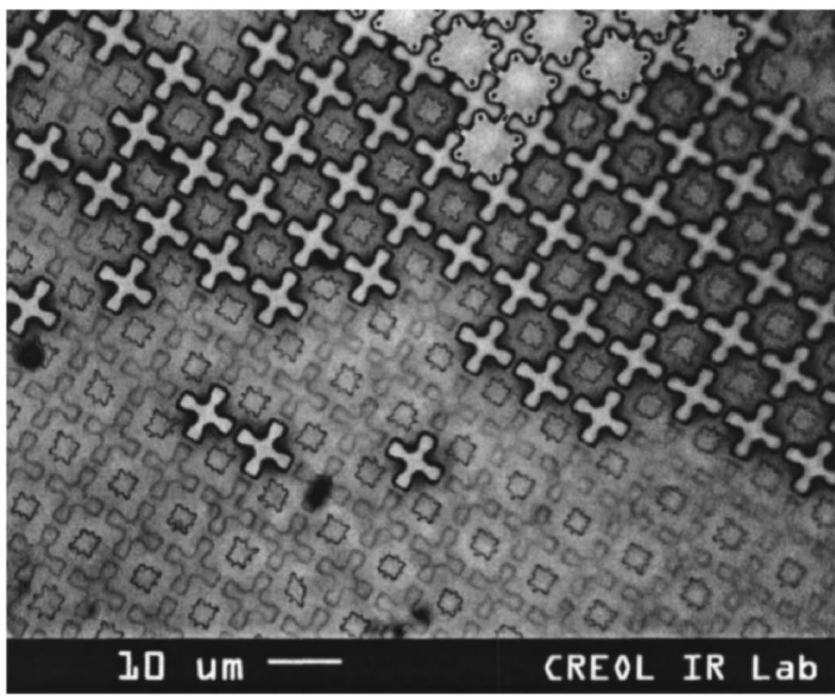

FIG. 3. PMGI lift-off results: bottom, ghost images left behind after disassociation of elements; middle, properly lifted off elements; top middle, elements before lift-off. 
sulted in the metal elements floating off the substrate along with the reacted portion of the PMGI leaving behind the ghost images seen in Fig. 3. Noble metals without an oxidation state at room temperature and standard pressure such as $\mathrm{Au}$ or $\mathrm{Ag}$ were considered to replace $\mathrm{Ti}$, but these nonreactive metals have no mechanism for adhesion.

Following the initial experiments with PMGI, we attempted to use a layer of silicon nitride or a layer of silica to insulate the Ti elements from the polymer layer. However, a mismatch in the thermal coefficients of the dielectrics compared to the polymer resulted in a cracking of the dielectric layer. This initially occurred when the substrate was cooling down after deposition of the dielectric, but the cracking became more pronounced following further thermal processing steps.

Of the three polymer standoff layer choices investigated in this study, BCB gave the most satisfactory results overall. Referring again to Fig. 2, we see that compared to the other polymers, BCB has the highest IR absorption. Although this is undesirable, the absorption is still low enough that it may be compensated for in the FSS design. BCB has an advantage over the other candidates because it has the ability, unlike ZEP, to form strong bonds with transition metals such as Ti. Unlike PMGI, the oxidation reaction between BCB and Ti does not result in the disassociation of the reacted product. Another advantage of $\mathrm{BCB}$ is that as a spin-on dielectric material, it can be thinned to achieve a layer of any thickness from $300 \mathrm{~nm}$ to $2 \mu \mathrm{m}$. It must, however, be cured at $250{ }^{\circ} \mathrm{C}$ in an inert environment. The cure temperature was not an issue for liquid polyimide films, which were not observed to deplanarize under these conditions.

In initial IR FSS fabrications, an optically thick $(150 \mathrm{~nm})$ $\mathrm{Au}$ film was used as the ground plane, which created a problem with the use of a polymer standoff layer because of the lack of adhesion between Au and BCB. During fabrication trials, it appeared that the metal elements were dissociating from the $\mathrm{BCB}$, but ellipsometric measurements proved that the BCB layer beneath the antenna elements was no longer present following the lift-off procedure. It was concluded that the BCB had insufficient adhesion with the Au ground plane. A solution was attempted by evaporating a Ti seed layer between the $\mathrm{Au}$ and the $\mathrm{BCB}$, but this was unsuccessful because the Ti did not adhere well to the Au.

$\mathrm{Au}$ adheres well to Ti because the surface of the Ti film is rough and provides a means for the polycrystalline $\mathrm{Au}$ to form a diffusive bond with the Ti. The Au film is smooth and does not provide an opportunity for the Ti film to form a strong bond. BCB forms a strong bond with Ti via oxidation. For this reason, subsequent FSS designs used an all-Ti ground plane. Figure 4 shows the spectral conductivity for $\mathrm{Au}$ and $\mathrm{Ti}$ as well as the spectral skin depth for both metals. At a wavelength of $10 \mu \mathrm{m}$, the skin depth of Ti is about $50 \mathrm{~nm}$ and the skin depth of $\mathrm{Au}$ is about $12 \mathrm{~nm}$. Either $150 \mathrm{~nm}$ thick metal film is sufficient to prevent transmission, but it is also desirable in the FSS design to have the highest possible spectral conductivity in the mid-IR range. Although $\mathrm{Au}$ is preferred for this reason, Ti may be used with appro-

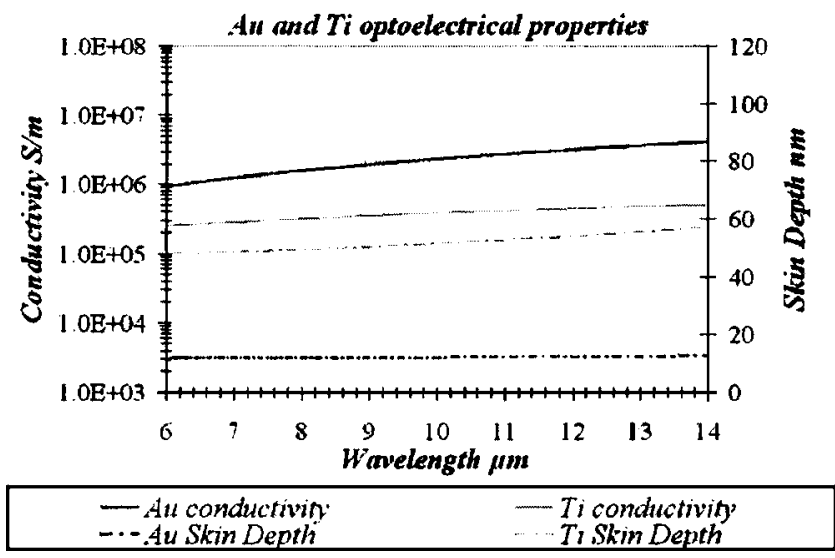

FIG. 4. Spectral conductivity and skin depth derived from optical constants measured with IR-VASE ellipsometer.

priate design compensation. Modeling software such as ANSOFT HFSS has been successfully modified to account for this spectral dependence. ${ }^{7}$

\section{FABRICATION RESULTS}

Three separate masks were designed consisting of a $10 \mathrm{~cm}$ (4 in.) square fully populated with FSS elements. Two different types of elements were used depending on the design. Two designs used Jerusalem cross elements as shown in Fig. 1, one with a $12.7 \mu \mathrm{m}$ periodicity and a $1.7 \mu \mathrm{m}$ critical dimension and the other with a $7 \mu \mathrm{m}$ periodicity and a $1.3 \mu \mathrm{m}$ critical dimension. The third type of element fabricated was a square-loop design ${ }^{1}$ with a $10 \mu \mathrm{m}$ periodicity and a $1.2 \mu \mathrm{m}$ critical dimension.

Fabrication of the FSS periodic structures used HD Microsystems liquid polyimide, which served as a structural substrate beneath the ground plane shown in Fig. 1. Liquid polyimide could be spun on and cured by the user. This allowed for control over surface roughness. HD Microsystems liquid polyimide was spun onto a prime-grade $\mathrm{Si}$ wafer at $2000 \mathrm{rpm}$. Each layer was $13 \mu \mathrm{m}$ thick and three layers were spun for a total substrate thickness of about $40 \mu \mathrm{m}$. The polyimide film was soft cured at $150{ }^{\circ} \mathrm{C}$ between each layer, and the final three-layer film was hard cured at $300{ }^{\circ} \mathrm{C}$ in an inert environment. The surface roughness was measured to be $4 \mathrm{~nm}$ rms with Dektak profilometry.

The remaining process steps were carried out as they would have been if the substrate was simply a Si wafer. The $150 \mathrm{~nm}$ thick Ti ground plane was deposited onto the liquid polyimide surface using an electron-beam evaporation system. Next, BCB was spun onto the Ti at a speed and viscosity combination appropriate to the desired thickness. Undiluted $\mathrm{BCB}$ was spun at $1500 \mathrm{rpm}$ to achieve a thickness of $1.8 \mu \mathrm{m}$. Charts for BCB showing the film thickness as a function of spin speed for given viscosities may be obtained from the Dow Corporation. BCB also uses an adhesion promoter (Dow AP3000) that goes on before the polymer film. The BCB film was soft baked at $120^{\circ} \mathrm{C}$ and hard cured in an inert environment at $250{ }^{\circ} \mathrm{C}$. 


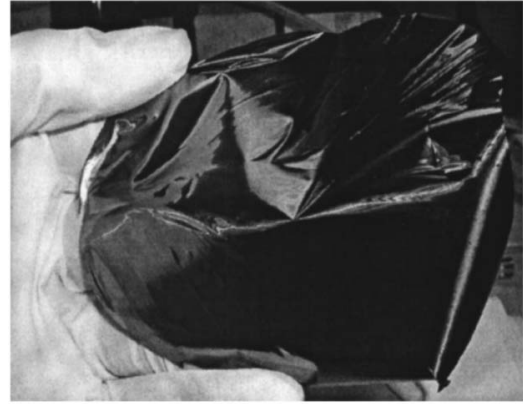

(a)

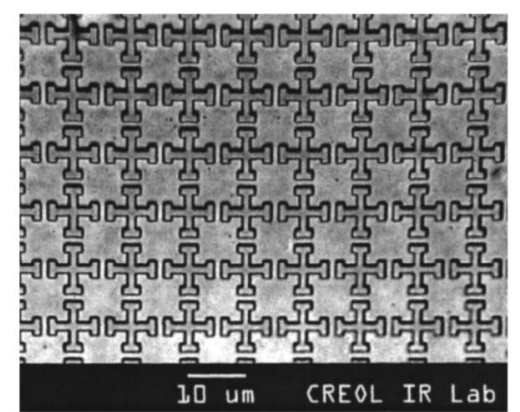

(b)
FIG. 5. Completed polyimide FSS: (a) flexible $10 \mathrm{~cm}$ (4 in.) wafer fully populated and (b) elements intact on flexible substrate after polyimide backing removal.
Electron-beam lithography was used to write the elements. This was done to ease fabrication issues associated with the resolution of the available photolithography systems. ZEP 520A7 was used as the electron-beam resist and cured for $3 \mathrm{~min}$ at $180{ }^{\circ} \mathrm{C}$. The liquid polyimide substrate led to nearly complete uniformity and well resolved elements across the populated region.

The final fabrication steps were to deposit a metal film onto the patterned resist. Depending on the FSS design, $100 \mathrm{~nm}$ of either Ti or Au was used for the antenna elements. A $10 \mathrm{~nm}$ thick $\mathrm{Ti}$ adhesion layer was used beneath the $\mathrm{Au}$ film. Both metals were deposited via electron-beam evaporation at $5.0 \times 10^{-6}$ Torr and $50{ }^{\circ} \mathrm{C}$. ZEP was removed by methylene chloride. Although lift-off was much faster with ZEP compared to standard photoresists, the wafer had to be quickly rinsed and reimmersed in solvent to prevent metal contaminants from settling on the surface.

Once the lithography processing was completed, the liquid polyimide could be peeled off the Si wafer. One way to peel the liquid polyimide off the substrate was to place a piece of weak adhesive tape across the surface and then pull the tape off. This did not remove the FSS structures and several repetitions would cause the polyimide to begin to separate from the substrate. Another method to remove the liquid polyimide from the Si wafer is to break the wafer and carefully peel the polyimide off as the two-halves of the wafer are pulled apart. Figure 5 shows the FSS after removal from the Si wafer. The metallized FSS elements are shown to be intact after removal in part (b) of Fig. 5.

The substrate flatness of the sample was measured using a Newton interferometer while the samples were still fixed to Si wafers. A transparent optical flat was placed in contact with the sample and a fringe pattern was observed using a mercury lamp. The lamp was filtered so that only the green line with $\lambda=546 \mathrm{~nm}$ was transmitted. Flatness was measured by counting the number of circular fringes per unit length. The liquid polyimide film on $\mathrm{Si}$ showed $0.06 \lambda / \mathrm{mm}$ in the center of the wafer and $0.08 \lambda / \mathrm{mm}$ toward the edges of the wafer. Deviations from flatness in the polyimide film were radially symmetric. This was likely a result of the spin-on application of the film. For comparison, a polyimide sheet was glued to a substrate and measured in the same manner. The polyimide sheet showed deviations in flatness ranging from 1.25 to $3.75 \lambda / \mathrm{mm}$.
The liquid polyimide FSS substrates were easily able to conform around a cylindrical axis, and there did not appear to be any damage to the FSS elements as the substrate was plastically deformed. ${ }^{8}$ The substrates are reasonably easy to handle but can be torn by a shearing force. They can handle a moderate degree of stretching along the plane of the structures. The liquid polyimide membranes may be used at temperatures up to $300{ }^{\circ} \mathrm{C}$. The fabrication techniques demonstrated in this article open the door for the fabrication of other structures such as IR detectors that may need to employ lithography on a similar element size.

\section{SUMMARY}

Three different periodic FSS structures were fabricated on liquid polyimide membranes. The periodic structures had periodicities from 1.2 to $1.7 \mu \mathrm{m}$ and fully populated a $10 \mathrm{~cm}$ (4 in.) diameter wafer. The FSS showed excellent uniformity across the wafer. The success of the fabrication suggests that the degree of nonflatness and surface roughness in these films was acceptable for 1-2 $\mu \mathrm{m}$ critical dimension lithography processes. The liquid polyimide structures had deviations in flatness from 0.06 to $0.08 \lambda / \mathrm{mm}$ and $4 \mathrm{~nm}$ rms surface roughness. BCB was used as an intermediate layer beneath the periodic structures. It was found to have sufficiently low absorption in the mid-IR as well as favorable adhesion properties for FSS fabrication. Two other polymers were found to have unfavorable adhesion properties. Au and Ti were used for the periodic structures as well as for the ground plane beneath the intermediate layer. The conductivities of $\mathrm{Au}$ and $\mathrm{Ti}$ at IR frequencies were measured, and $\mathrm{Ti}$ was found to be less conductive (by nearly an order of magnitude) than Au. The fabrication techniques described here would be suitable for the fabrication of many different types of periodic microstructures on flexible substrates.

\section{ACKNOWLEDGMENT}

A portion of this work was done at the Cornell Nanofabrication facility at Cornell University.

\footnotetext{
${ }^{1}$ B. Monacelli, J. Pryor, B. A. Munk, D. Kotter, and G. D. Boreman, IEEE Trans. Antennas Propag. 53, 745 (2005).

${ }^{2}$ B. A. Munk, Frequency Selective Surfaces, Theory and Design (Wiley, New York, 2000), pp. 26-62.

${ }^{3}$ A. Mahmood, D. P. Butler, and Z. Celik-Butler, IEEE Sensors 2005, 30
} 
October-3 November 2005, p. 4.

${ }^{4}$ R. P. Drupp, J. A. Bossard, D. H. Werner, and T. S. Mayer, IEEE Antennas and Propagation Society International Symposium 2004, 20-25 June 2004, Vol. 2, pp. 1907-1910.

${ }^{5}$ S. Young, D. Weston, B. Dauksher, D. Mancini, S. Pacheco, P. Zurcher, and M. Miller, J. Micromech. Microeng. 15, 1824 (2005).
${ }^{6}$ P. Bebin and R. Prud'homme, J. Polym. Sci., Part B: Polym. Phys. 40, 82 (2002).

${ }^{7}$ J. Ginn, B. Lail, D. Shelton, J. Tharp, W. Folks, and G. Boreman, Appl. Comput. Electromagn. Soc. J. 22, 184 (2007).

${ }^{8}$ N. Chen, J. Engel, S. Pandya, and C. Liu, MEMS 2006 Conference, Istanbul, Turkey, 22-26 January 2006. 